\title{
Análisis del grado de satisfacción de docentes de la Universidad Nacional de Pilar, con las condiciones en que trabajan a partir de indicadores intrínsecos y extrínsecos de satisfacción laboral
}

\author{
Nelly Ceferina Amarilla Sánchez \\ Universidad Nacional de Pilar \\ nelly_amarilla@hotmail.com
}

\section{RESUMEN}

La investigación tuvo por objeto analizar el grado de satisfacción de los docentes de la Universidad Nacional de Pilar con las condiciones laborales en que imparten su trabajo a partir de indicadores intrínsecos y extrínsecos de satisfacción laboral. La satisfacción fue abordada en términos de las recompensas intrínsecas y extrínsecas que experimentaron en su trabajo; las actividades de docencia, investigación y gestión que desempeñan; el apoyo y reconocimiento por su desempeño académico; así como la situación de las instalaciones y equipamientos de las diferentes unidades académicas. Con base en una investigación no experimental, de tipo descriptivo, corte transversal y enfoque cualicuantitativo, se recogieron datos a través de un cuestionario antes utilizado en varios contextos. El mismo se aplicó a 120 profesores catedráticos de cinco unidades académicas de la universidad. El grado de satisfacción general con los indicadores estudiados ha sido de bajo a medio, lo que implica la necesidad de un análisis profundo de las condiciones físicas y laborales donde los docentes ejercen su profesión si los directivos prevén una educación de calidad y la preparación de profesionales competentes.

Palabras clave: Satisfacción, docencia, investigación, gestión, condiciones laborales. 


\title{
Analysis of the degree of satisfaction of teachers at the National University of Pilar, with the conditions in which they work from intrinsic and extrinsic indicators of job satisfaction
}

\begin{abstract}
The purpose of the research was to analyze the degree of satisfaction of teachers at the Universidad Nacional de Pilar with the working conditions in which they teach their work, based on intrinsic and extrinsic indicators of job satisfaction. Satisfaction was addressed in terms of the intrinsic and extrinsic rewards they experienced in their work; the teaching, research and management activities they carry out; support and recognition for their academic performance; as well as the situation of the facilities and equipment of the different academic units. Based on a non-experimental, descriptive, cross-sectional and qualitative-quantitative approach, data was collected through a questionnaire previously used in various contexts. It was applied to 120 university professors from five academic units of the university. The general degree of satisfaction with the indicators studied has been low to medium, which implies the need for an in-depth analysis of the physical and working conditions where teachers practice their profession if the managers provide for a quality education and the preparation of professionals. competent.
\end{abstract}

Keywords: Satisfaction, teaching, research, management, working conditions.

Artículo recibido: 02. abril. 2020

Aceptado para publicación: 11. junio. 2020

Correspondencia: nelly_amarilla@hotmail.com

Conflictos de Interés: Ninguna que declarar 


\section{INTRODUCCIÓN}

La satisfacción laboral es probablemente la actitud ante el trabajo más estudiada desde que empezó a ser considerada explícitamente en los años treinta como parte esencial del comportamiento humano en ambientes de trabajo (Brief, 1998; Locke, 1976 citado por Galaz Fontes, 2003). Puede ser analizada como un concepto global y por tanto unidimensional, o bien, como un concepto multidimensional considerando diferentes aspectos o facetas del trabajo que pueden variar de forma independiente. Éstas suelen estar relacionadas con aspectos tales como las características del puesto de trabajo, la compensación económica, las oportunidades de promoción, las relaciones con el jefe y con los compañeros (Smith et al., 1969 citado por Capelleras, 2001).

La satisfacción en el trabajo es el resultado de varias actitudes que tiene un empleado hacia su trabajo, los factores conexos y la vida en general, (Blum y Nayles, 1995 citado por Murillo, 2006); se entiende como satisfacción individual en una relación laboral dentro de una empresa -universidad- (Mankeliunas,1996). Es la sensación de bienestar derivada de las condiciones de trabajo, de la realización de las tareas, de la pertenencia a la organización y de conseguir objetivos y logros profesionales (Martínez Selva, 2004).

Koontz y O’Donell (1995), plantean que la satisfacción laboral se refiere al bienestar que se experimenta en el trabajo, cuando un deseo es satisfecho, relacionándolo también con la motivación en el trabajo. Coincidiendo al respecto con Chiavenato (1986), al señalar que la satisfacción en el trabajo designa la actitud general del individuo hacia su trabajo (citados por Murillo, 2006).

La satisfacción en el trabajo se entiende como como resultado de lo que se hace; en este caso se clasifican las funciones parciales en tres grupos (Mankeliunas, 1996):

a) Satisfacción interna, abarca la actitud interna del trabajador frente al trabajo que realiza, la satisfacción con el producto, el poder de perfeccionar los pasos del proceso.

b) Satisfacción concomitante, consta de las condiciones materiales y ambientales de los compañeros y mandos medios y superiores, que acompañan al proceso de trabajo.

c) Satisfacción externa, provocada por la remuneración que satisface las necesidades del trabajador. 
En cuanto a la importancia, la política de recursos humanos de la empresa es decisiva para la satisfacción laboral en múltiples facetas, atendiendo los ámbitos en que se origina; estilo de dirección, comportamiento de grupos pequeños (microclimas), el entorno profesional relacionado con las tareas y responsabilidades desempeñadas (Martínez Selva, 2004).

La satisfacción laboral está ligada al desarrollo de trabajadores como personas y a su dignidad, en tanto se relaciona con la calidad de vida en general; y, porque un empleado satisfecho presentará más conductas en favor de la organización que uno menos satisfecho (Kalleberg, 1977 citado por Galaz Fontes, 2003).

En un período de gran dinámica tecnológica y económica, las organizaciones se deben adaptar continuamente a sus ambientes y fomentar las condiciones que garantizan la existencia de trabajadores satisfechos; lo que en definitiva garantiza ganancias económicas significativas para la organización.

Trasladando lo expuesto al ámbito universitario, Zabalza (2004) declara que la mejora del nivel de satisfacción de los profesores hará posible la dinamización institucional de los centros universitarios y la mejora de la calidad de los procesos formativos que en ellos se producen.

La descripción de los hechos acerca de la satisfacción en el trabajo es muy uniforme, pero, al interpretarlos, los investigadores sugieren una gran variedad de explicaciones (Mankeliunas, 1996). Una de ellas, es la clasificación iniciada por K. B. Madsen (1980 citado por Mankeliunas, 1996), que divide a estas teorías en cuatro grupos: las basadas en la satisfacción de necesidades, las que parten de la concepción del incentivo, las concepciones cognoscitivas de la satisfacción y las humanísticas de la satisfacción.

\section{Teorías basadas en la satisfacción de necesidades}

Estas teorías se basan en el supuesto de que cada persona tiene una serie de necesidades primarias y secundarias (aprendidas) que busca satisfacer, es decir, busca un equilibrio (homeostasis) y cuando éste se pierde trata de reconstruirlo. Cuando una persona no satisface sus necesidades pierde este equilibrio y en seguida se presenta una activación interna que busca recuperarlo, a fin de que el individuo tenga una representación interna de satisfacción. 
Si se aplica esta teoría a la situación del trabajo, la persona labura para alcanzar medios que satisfagan sus necesidades primarias y, luego, para cubrir sus necesidades sociales. La mayoría de estas teorías interpretativas de la satisfacción en el trabajo se basan en las cinco necesidades de Maslow: seguridad, contacto social, reconocimiento, independencia personal y autorrealización. Esta interpretación se funda en la valoración de las situaciones ideal y real. Con base en esta interpretación, la satisfacción en el trabajo se puede representar de la siguiente manera: Satisfacción en el trabajo = Valor ideal Valor real. Cuando la diferencia entre los valores ideal y real es cero, existe satisfacción; cualquier otro caso indica insatisfacción (Mankeliunas, 1996).

\section{Teorías basadas en la concepción del incentivo}

En las teorías basadas en el incentivo predomina el principio del placer y el displacer, el gusto y el disgusto. Estas teorías se distinguen de las anteriores porque afirman que el individuo no persigue la satisfacción de alguna de sus necesidades, sino que busca maximizar su placer mediante los incentivos del ambiente. Para algunos autores, esta situación es característica de las sociedades de consumo. Mankeliunas (1996).

\section{Concepciones cognoscitivas de la satisfacción}

Siguiendo con el análisis de Mankeliunas (1996), las concepciones cognoscitivas en el proceso motivacional consideran que la persona tiene la capacidad de reconocer su situación actual concreta y representársela en otras condiciones, hacia las que puede tender su acción concreta. La persona es capaz de prever las posibilidades de salir de una situación real por medio de un plan de actividad, tendiente a cambiar el estado actual. En la situación laboral, el trabajador puede no estar satisfecho con su posición actual, pero elabora un plan que por intermedio de su propia actividad y dirigiendo la de otras personas, puede llegar a una nueva situación que puede proporcionar estados satisfactorios.

En otras palabras, el hombre conoce la situación real concreta, prevé soluciones reales posibles y se pone en acción para alcanzarlas; es decir, primero trabaja conceptualmente y luego pone en práctica sus conclusiones.

Aun cuando en la práctica no son muchos los autores que generalizan sus datos acerca de la satisfacción en el trabajo a partir del constructo cognoscitivo de la motivación, actualmente se tiende más hacia esta concepción. La satisfacción laboral se 
inicia con la concepción de incentivo, pero sus expectativas prolongan los incentivos concretos hacia el futuro, en el que se satisfarán mejor las posibilidades personales (Mankeliunas, 1996).

\section{Concepción humanista de la satisfacción}

En su análisis, Mankeliunas (1996) destaca que son dos factores los que han engendrado la concepción humanista de la satisfacción en el trabajo: la tendencia en la industria a humanizar el trabajo y la aparición de la psicología humanista.

En cuanto a la satisfacción en el trabajo, los autores humanistas tomaron muchos elementos de la teoría de Maslow sobre la motivación en general y aplicaron conceptos básicos a la satisfacción en las situaciones laborales, especialmente F. H. Herzberg y su equipo.

La concepción de la motivación de Maslow está orientada hacia la satisfacción de necesidades, pero distingue dos clases de motivos: deficitarios y de crecimiento. Sólo para la primera clase de motivos es válida la explicación basada en el equilibrio, la insatisfacción de alguna necesidad induce a la persona a buscar comportamientos instrumentales para satisfacerla. La insatisfacción de esta clase de necesidades conduce a la enfermedad. La satisfacción de necesidades biológicas evita enfermedades, pero no asegura el estado de salud; por eso, el hombre necesita de motivos de crecimiento (motivos humanos y sociales) que nunca conducen al estado ideal, sino que sólo indican progreso en cuanto a estos ideales. Un hombre que busca su autorrealización se caracteriza por la espontaneidad, independencia, comunicación social, etc. El estado de satisfacción no significa un estado de inercia, sino una continua creatividad al servicio de la comunidad. Por consiguiente, la satisfacción en el trabajo es sólo una parte de este desarrollo espontáneo de las disposiciones y actividad del individuo (Mankeliunas, 1996).

A partir de la propuesta de Maslow acerca de la diferenciación y jerarquización de las necesidades, Herzberg propuso el todavía muy influyente concepto de motivaciónhigiene o teoría de dos factores. Según Herzberg, subraya Galaz Fontes (2003), la satisfacción con el trabajo es esencialmente un estado emocional asociado con el trabajo de uno, derivado de la interacción de dos variables independientes; la satisfacción con el trabajo y su insatisfacción. La satisfacción laboral toma lugar como una reacción a la presencia de cinco "motivadores" (factores de contenido de trabajo) asociados a la naturaleza del trabajo en sí mismo o el logro, el reconocimiento por el trabajo cumplido, 
el trabajo en sí, la responsabilidad, y las oportunidades para el desarrollo del individuo. Todos estos factores tienen en común su relación con las necesidades de crecimiento y auto-realización de las personas.

Los modelos de satisfacción laboral sustentados en las necesidades y valores sostienen que: a) los individuos tienen deseos o necesidades por varios tipos de resultados; b) los ambientes de trabajo proporcionan a los individuos niveles diferentes de tales resultados; y c) los estados afectivos de los individuos son determinados en gran medida por la correspondencia entre los niveles deseados y los obtenidos en cuanto a los resultados (Stone, 1992 citado por Galaz Fontes, 2003).

Galaz Fontes (2003) señala a Locke (1976) quien identifica tres tendencias históricas o escuelas en la identificación de los factores considerados causantes de la satisfacción con el trabajo: el físico-económico, las relaciones sociales o humanas y el trabajo per se como un medio de desarrollo humano. Estas tres posiciones, analiza el mencionado autor, asumen que la satisfacción en el trabajo es una consecuencia directa de ciertas variables y no suponen conceptualizaciones teóricas adicionales a la identificación de los aspectos relevantes del trabajo, considerados responsables de fomentar la satisfacción laboral entre los empleados.

\section{METODOLOGÍA}

El estudio se ajusta a un diseño no experimental, de corte transeccional y tipo descriptivo. Conforme lo señala Hernández Sampieri et al (2008), la investigación no experimental se realiza sin manipular deliberadamente variables, sino más bien, se observan los fenómenos tal como se dan en su contexto natural, para después analizarlos. El desarrollo del estudio tuvo por objeto analizar el grado de satisfacción de los docentes de la UNP con las condiciones en que trabajan a partir de indicadores intrínsecos y extrínsecos de satisfacción laboral. Con ello, se pretendió presentar la realidad tal cual es en el ámbito laboral. El estudio es de corte transeccional (Hernández Sampieri et al (2008), en el cual los datos se recolectan en un solo momento. Finalmente, la investigación es un estudio descriptivo con sujetos activos en la UNP, que se desempeñan en el ámbito académico. Los estudios descriptivos, según Hernández Sampieri et al (2008), tienen como propósito presentar el fenómeno estudiado, así como aparece en el contexto particular, sin la relación o manipulación de variables, conllevando en sí el único 
propósito de constatar hechos como existen en la realidad. Es importante destacar que un estudio descriptivo persigue, según H. Sampieri et al. (2006), contrastar hipótesis, más bien que comprobar relación entre variables.

El enfoque empleado fue la mixta, cualitativo y cuantitativo. El abordaje cualitativo ayuda en la interpretación, comprensión y generación de teoría a partir de los resultados logrados; en tanto que, la recolección, procesamiento y análisis de los datos numéricos de las variables se realizó en base al método cuantitativo.

La población del estudio estuvo conformada por 172 docentes catedráticos de cinco Unidades Académicas de la UNP sede en Pilar, excluyendo las distintas filiales. La investigación se desarrolló en el período académico 2010 con una muestra no aleatoria intencional de 120 docentes, asistentes en su mayoría y sin cargos administrativos.

La técnica de recolección de datos fue la encuesta; se utilizó un cuestionario de escala de calificación numérica validado por Veciana y Capelleras (2001) en un estudio sobre la Calidad y Satisfacción del Profesorado con la Enseñanza y su Puesto de Trabajo en la Universidad de Barcelona, también adaptado por Rosales (2005) en un estudio sobre Percepción de la Calidad de la Enseñanza desde la Perspectiva de sus Actores (Tesis Doctoral - Chile), y por Benítez y Del Puerto (2008) en un estudio para conocer el Nivel de Satisfacción de los Docentes del Nivel Medio de Colegios Nacionales de la Ciudad de San Ignacio Misiones - Paraguay, Tesis para acceder al Grado Académico de Magíster por la Universidad de Viña del Mar - Chile.

\section{Contextualización}

La Universidad de Pilar se constituyó un 14 de julio de 1.991, fue reconocida como Universidad Nacional por Ley de la Nación, el 28 de diciembre de 1994. Hoy, a casi 10 años de la presente investigación, se pretende dar a conocer los resultados obtenidos como trabajo de tesis doctoral y darle una continuidad en recoger nuevos datos en el 2020, de modo a contrastar los resultados. En el año 2010, al momento de la recolección de datos, la universidad contaba con cinco Facultades, una Escuela de Postgrado, así como la Escuela Básica "Dr. Narciso González Romero", y sedes en las ciudades de San Ignacio, San Juan Bautista y Ayolas, del departamento de Misiones. Se aclara que, en la actualidad, visiblemente los resultados serían superados, pues la institución fue creciendo de un modo acelerado, aumentando la cantidad de alumnos y de profesionales docentes. 
La institución está asentada en la ciudad de Pilar, capital del $12^{\circ}$ departamento, Ñeembucú, distante a unos 360Km. de la ciudad de Asunción. El departamento cuenta con una superficie aproximada de $12.147 \mathrm{Km} 2$., y una población cercana a los 80.000 habitantes, representa el 3\% del área total del país y el 1,5\% de la población total, localizado en el vértice Suroeste de la Región Oriental del Paraguay, limitando en esa dirección con la República Argentina por medio de los Ríos Paraguay y Paraná, al norte con los departamentos Central y Paraguarí y al este con el de Misiones. Se estima que entre el 30 y $50 \%$ de su territorio se encuentra permanentemente bajo agua la mayor parte del año, constituyendo importantes humedales y áreas naturales que deben preservarse; asimismo es considerado como una de las regiones más ricas en cuanto hace a la historia nacional, por los escenarios principales en la Guerra Grande (1864-1870).

\section{RESULTADOS Y DISCUSIÓN}

Los tiempos actuales nos obligan a repensar la Universidad del siglo XXI; a pesar de que la formación de profesionales, la investigación y la extensión a la comunidad siguen siendo funciones principales de la misma. Interesa a la investigación las actitudes de los docentes con el objeto de detectar si sienten o no satisfacción en su trabajo y ver en qué forma se podrían visualizar aquellos factores que están influyendo en dicha satisfacción, considerando la gran importancia que tiene el docente como uno de los actores responsables del ambiente o clima social del aula; situación que exige una actitud positiva de los mismos en el desempeño de sus funciones para responder con eficiencia y eficacia a los nuevos requerimientos sociales.

Para identificar las recompensas intrínsecas que reciben los catedráticos de la Universidad Nacional de Pilar se recogieron datos relacionados con la sensación de bienestar que experimentan en cuanto a autonomía laboral, impacto en otras personas externas al Proceso de Enseñanza y Aprendizaje (PEA), sentimiento de realización personal en el trabajo, oportunidad para el aprendizaje continuo, y oportunidad para utilizar las propias habilidades en el trabajo. Los datos reflejan claramente que los docentes de la Universidad Nacional de Pilar se sienten satisfechos respecto a esta variable y para ahondar más en el análisis se establecieron algunos cruzamientos entre los indicadores, los cuales permitieron visualizar la relación existente entre los mismos. 
Gráfico 1. Nivel de satisfacción de los profesores con las recompensas intrínsecas.

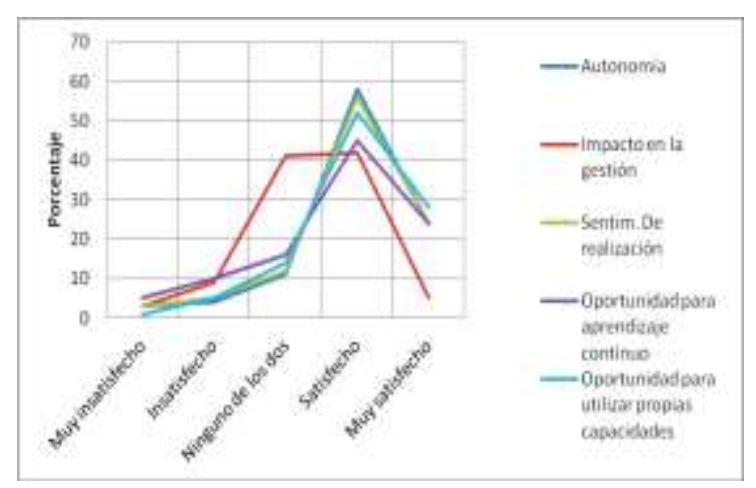

Con ello se confirma la hipótesis de trabajo "las recompensas de carácter intrínseco que ofrece el puesto de trabajo a los docentes de la Universidad Nacional de Pilar constituyen el aspecto más importante de satisfacción laboral”.

Respecto al nivel de satisfacción que experimentan los profesores catedráticos, por el impacto que tienen sus gestiones en las personas externas a su gestión, manifestaron estar ni satisfechos ni insatisfechos, muy próximo a lo que reportaron sí estar satisfechos por el impacto que tiene o pueda tener sus gestiones en otras personas que no fueran sus alumnos. Este dato que expresa indecisión, e incluso apatía o indiferencia, debe formar parte de la gestión de la dirigencia para exhibir un mayor compromiso de los estamentos encargados.

Se han realizado cursos de formación continua de profesores, que no se conforman con el título de grado y están abocados a la realización de cursos de postgraduación; por cuanto los niveles altos de satisfacción requieren el acompañamiento institucional y el establecimiento de una política de recursos humanos, que es decisiva para la satisfacción laboral en múltiples facetas. Los datos obtenidos permiten visualizar que los informantes están satisfechos con las oportunidades de aprendizaje continuo, lo cual es significativo, ya que un empleado satisfecho demostraría un mayor compromiso y una mejor performance en su trabajo.

La mayoría de los docentes se sienten satisfechos respecto a las oportunidades para utilizar sus capacidades en el desarrollo de sus actividades docentes, siendo un aliciente fundamental para potenciar la mejora educativa en la institución. Así, pues, los datos obtenidos son elocuentes, y exigen que desde la institución se promueva el trabajo como desarrollo personal, dando énfasis a la habilidad, la eficacia y la responsabilidad en las tareas mentalmente desafiantes, como diría Galaz Fontes en atención a los factores más influyentes en la satisfacción laboral. 
Para identificar las recompensas extrínsecas que obtienen los profesores de la Universidad Nacional de Pilar por el trabajo que realizan, se recopilaron datos relacionados con el salario, los beneficios extra-salariales, las becas para continuar estudiando, la seguridad en el puesto de trabajo y las oportunidades de promoción. La mayoría respondió que tales recompensas les son indiferentes, no tienen definición si están o no satisfechos. El $43 \%$ están satisfechos con el salario. Ninguna variable alcanza el 50\% de satisfacción, indicando que nadie aparentemente está muy satisfecho. Es decir, resalta los niveles más altos de insatisfacción docente.

Gráfico 2. Niveles distintos de satisfacción sobre las recompensas extrínsecas.

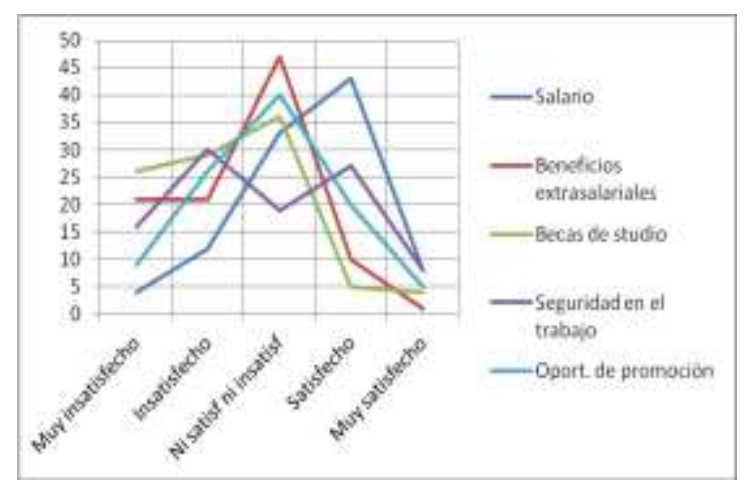

Con esto, se comprueba la hipótesis que expresa "las recompensas de carácter extrínseco que ofrece el puesto de trabajo a los docentes de la Universidad Nacional de Pilar constituyen las principales fuentes de insatisfacción laboral”.

Cabe mencionar, que la satisfacción laboral se inicia con la concepción de incentivo, pero sus expectativas superan el simple nivel de incentivos concretos y se proyectan hacia el futuro, siempre y cuando las posibilidades personales son satisfechas; y esto a su vez permite la mejora en el rendimiento. Por lo mismo, en el trabajo se analizan más específicamente los resultados obtenidos para identificar debidamente dónde se producen los niveles más altos y más bajos de satisfacción, contrastando con la edad, el género, la situación laboral, la antigüedad y el grado académico de los mismos.

Referente a las actividades de docencia, investigación y gestión que desempeñan los docentes catedráticos de la UNP, se sigue el mismo procedimiento que los demás objetivos. Los resultados obtenidos indican que los profesores se encuentran levemente más satisfechos por la actividad docente que desempeñan en la institución en estudio, que respecto a la actividad de investigación que desempeñan, la media es de ni satisfecho ni insatisfecho, al igual que las actividades de gestión en general. 
Las causas de la insatisfacción no fueron encaradas en este estudio; sin embargo, es una problemática que bien podría ser objeto de otra investigación, de modo a resolver el origen de la misma y encauzar estrategias de mejora. Cabe mencionar, que recién unos años atrás pudo lograrse la incorporación del área de investigación en la designación presupuestaria, quizás sea uno de los motivos que determinan el nivel de satisfacción pero que deberá dilucidarse con otra investigación. Asimismo, en cuanto a la gestión, pues la tendencia de insatisfacción es muy alta en todas las variables intervinientes.

Gráfico 3. Actividades de docencia, investigación y gestión que desempeñan.

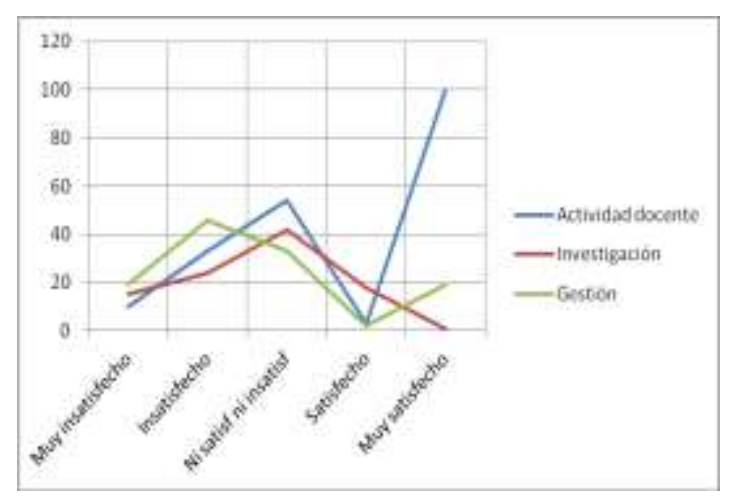

Los docentes de la Universidad Nacional de Pilar, se encuentran satisfechos por las actividades de docencia e investigación y en menor grado por las gestiones, coincidentes con la hipótesis planteada "La enseñanza y la investigación constituyen las actividades más satisfactorias que desempeñan los docentes de la Universidad Nacional de Pilar; sin embargo, las tareas de gestión son las más insatisfactorias”.

Por otra parte, para detectar el apoyo y el reconocimiento que los docentes reciben por el desempeño académico en su disciplina científica, se trabajó a partir de los siguientes indicadores: relación con los compañeros de trabajo, apoyo de la dirección y autoridades universitarias, reconocimiento por su trabajo en la disciplina que enseña.

Los profesores de la Universidad Nacional de Pilar se hallan satisfechos respecto a las relaciones con los compañeros de trabajo, valor muy importante para el trabajo armónico en la institución por lo que las autoridades y los mismos docentes deben resguardar las buenas relaciones que mantienen entre sí los colegas, a pesar que existe un alto porcentaje de docentes que respondieron con la opción ni satisfecho ni insatisfecho. Respecto al apoyo de la dirección y autoridades universitarias, un buen número de docentes de la población en estudio prefirió la opción ni satisfecho ni insatisfecho, a más de insatisfechos; asimismo, los docentes reclaman mayor reconocimiento por su trabajo 
en la disciplina que enseñan; lo que constituye un margen elevado que debe llamar la atención de los directivos, reflexionar y actuar en post de la mejora.

De todos modos, este punto constituye una fortaleza que la institución debe fructificar con la convivencia administrativa y académica; pues los datos revelan además que la mayoría de los profesores, independiente a los años de servicios que tengan en la universidad en estudio, a la categoría docente y al grado académico que ostentan, se encuentran satisfechos por las relaciones que mantienen con sus compañeros de trabajo.

Gráfico 4. Apoyo y reconocimiento que los docentes reciben por el desempeño académico en su disciplina científica.

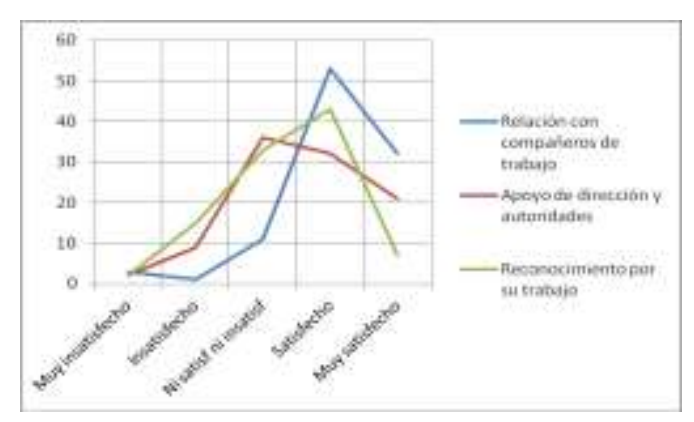

La hipótesis respecto a las relaciones con los compañeros queda rechazada, pues los docentes se hallan satisfechos, y queda comprobada en cuanto a: "El nivel de satisfacción de los docentes de la Universidad Nacional de Pilar es bajo en relación ... al apoyo recibido de la dirección académica y al reconocimiento del trabajo académico en su disciplina cientifica".

Finalmente, para el análisis del objetivo referente a las instalaciones y equipamientos de las diferentes unidades académicas se recopilaron datos concernientes a las condiciones de la sala de clases, equipamientos (laboratorios, computadoras, etc.), bibliotecas e instalaciones físicas en general. Con respecto a la valoración que hacen los profesores de las condiciones de las salas de clases, del equipamiento como ser laboratorios, computadoras, entre otros, la media de los datos se ubica en ni satisfecho ni insatisfecho; en cuanto a la bibliografía disponible la media de los valores obtenidos se encuentra en insatisfecho, y respecto a las instalaciones físicas de las distintas unidades académicas donde trabajan, la media de los valores obtenidos se ubica en ni satisfecho ni insatisfecho. Resultados más que elocuentes, que exigen una rápida atención para permitir un espacio más agradable y saludable para el desarrollo eficiente y eficaz del proceso de enseñanza y aprendizaje. 
Gráfico 5. Instalaciones y equipamientos de las distintas unidades académicas.

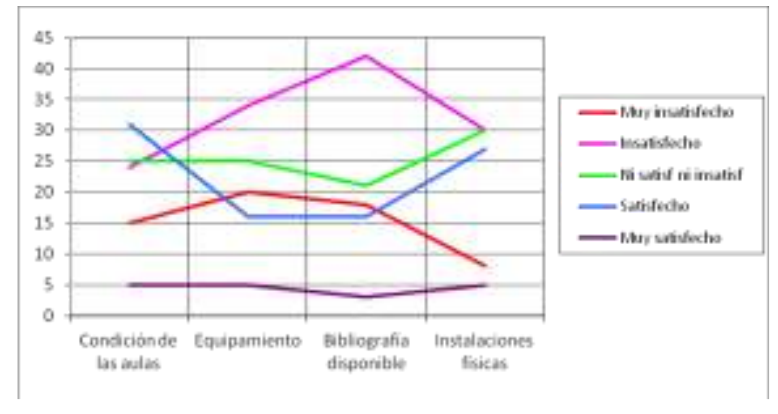

De este modo queda comprobada la hipótesis referente a "las condiciones de las aulas de clases, el equipamiento, las bibliografías disponibles y las instalaciones físicas en general tienden a generar insatisfacción en los docentes de la Universidad Nacional de Pilar”.

La mayoría de los profesores se encuentran insatisfechos por las condiciones de las instalaciones físicas en las que trabajan y con el equipamiento con que cuentan en la Universidad Nacional de Pilar, agravándose aún más cuando se trata de la bibliografía disponible en la institución y equipamientos existentes y a los cuales tienen acceso los docentes para desarrollar sus actividades académicas, en coherencia a los factores ambientales del trabajo propuesto por Herzberg y a la satisfacción concomitante de Mankeliunas.

Del análisis de los resultados se deduce que, en general, los docentes no están satisfechos con las condiciones físicas y con los recursos de aprendizaje disponibles en la UNP, su lugar de trabajo. Está comprobado en todas las sociedades que para que la actividad laboral sea efectiva y el ejecutor eficiente, ya sea en la producción de insumos o en servicios diferentes, el trabajador necesita de un lugar saludable, de los recursos físicos y humanos adecuados y de condiciones apropiadas para un buen desempeño y una labor de calidad.

\section{REFLEXIONES FINALES}

Se concluye que el escenario está apto para la concreción de los objetivos institucionales, pues se evidencia una concepción cognitiva y humanista de la satisfacción del personal docente de la universidad. Atendiendo que en cuanto el trabajo humano tiene un sentido para la realización de cada individuo; el trabajador busca su autorrealización, para lo cual necesita de las condiciones para lograrlo, que la dirigencia debe considerar. Esto confirmado con el grado satisfactorio alcanzado en los resultados de la variable recompensas intrínsecas. 
Sin embargo, respecto a las recompensas extrínsecas se detectó que constituyen los niveles más altos de insatisfacción docente. Por cuanto, debe atenderse a la concepción de incentivos, para posibilitar la satisfacción personal del docente, y por ende, garantizar la mejora en el rendimiento.

En cuanto a las actividades de docencia y la investigación se encuentran satisfechos; asimismo se visualiza un gran margen de "ni satisfecho ni insatisfecho"; en menor grado respecto a la gestión. Cabe mencionar, que las causas de la insatisfacción no fueron encaradas en este trabajo.

En atención a la implementación del enfoque cognitivo y de desarrollo personal asumido por la institución, es menester la especial atención a la concienciación de todos los actores involucrados en el logro de competencias y capacidades, a través de la implementación de estrategias que permitan valorar las actividades de docencia, investigación y gestión que desempeñan los docentes catedráticos de la UNP. El abordaje de los altos índices de insatisfacción o grado de ni satisfechos ni insatisfechos requiere concienciación y preparación hacia el desarrollo de la investigación.

Respecto al apoyo y reconocimiento, resalta que los docentes reclaman mayor reconocimiento por su trabajo en su disciplina científica, lo que constituye un margen elevado que debe llamar la atención de los directivos, reflexionar y actuar en post de la mejora. En cambio, se percibe un alto grado de satisfacción respecto a las relaciones que mantienen los docentes entre sí; debiendo considerarse este punto como una fortaleza que los directivos y propios docentes deben afianzar.

De los resultados se deduce que los docentes no están satisfechos con las condiciones físicas y con los recursos de aprendizaje disponibles en la UNP; siendo unos de los niveles más altos de insatisfacción los equipamientos y materiales bibliográficos disponibles. Por cuanto, es imprescindible que las autoridades y actores de la educación presten real atención a los indicadores de bajo nivel de satisfacción, a más de fortalecer aquellos indicadores que dan satisfacción al personal docente, para posibilitar la mejora de la calidad educativa; si bien, es sabido que como institución pública depende en gran medida de la asignación presupuestaria.

Se recomienda a los directivos de la Institución en estudio; proseguir con los cursos de formación y capacitación continua, para potenciar el desarrollo de la actividad académica y reconocer el esfuerzo de aquellos docentes que se sienten comprometidos y 
demuestran pertinencia institucional, a través de la concreción del escalafón docente y la estabilidad laboral. Al apoyar la formación técnico-pedagógica de los docentes, ofreciendo becas e intercambios docentes, nacionales e internacionales, y en atención al logro de las recompensas intrínsecas se obtendrá mejores resultados de satisfacción laboral.

Es menester, prestar especial atención a las recompensas extrínsecas, implementando mejoras, especialmente en lo concerniente a beneficios extrasalariales, becas para proseguir estudios y seguridad en el puesto de trabajo.

Para la mejora de la infraestructura y equipamiento de las facultades, es preciso gerenciar y concretar proyectos, acuerdos y convenios interinstitucionales, de modo a revertir la falta de inclusión en el presupuesto general de gastos de la nación.

Se recomienda a los docentes, que asuman desde su rol el compromiso de la mejora educativa, afianzando su sentido de pertinencia institucional y participando activamente de las actividades de la Universidad Nacional de Pilar, para permitir el crecimiento no sólo de la institución, sino también para lograr el desarrollo personal y profesional de cada uno y por ende, la instauración de la cultura universitaria en el contexto local y regional.

\section{LISTA DE REFERENCIAS}

Capelleras I Segura, Joan-Lluís. Factores condicionantes de la calidad de la enseñanza universitaria: un análisis empírico. Universidad Autónoma de Barcelona (tesis doctoral) [fecha de consulta: 9 de junio de 2009] Disponible en: http://www.tesis enxarxa.net/TESIS_UAB/AVAILABLE/TDX-0125102-101659//jlcs1de2.pdf

Chiavenato, Idalberto. (1992). Administración de Recursos Humanos. $2^{\circ}$ Edición. Bogotá. Editorial Mc Graw Hill.

Chiavenato, Idalberto. (1994). Introducción a la Teoría General de la Administración. $3^{\circ}$ Edición. Bogotá. Editorial Mc Graw Hill.

DECLARACIÓN MUNDIAL SOBRE LA EDUCACIÓN SUPERIOR EN EL SIGLO XXI: visión y acción [15 de junio de 2009] Disponible en: www.unesco.org/education/educprog/wche/declaration_spa.htm

Definición de apoyo. [fecha de consulta: 22 de marzo de 2010] Disponible en: http://www.definicionabc.com/general/apoyo.php

Definición de reconocimiento. [fecha de consulta: 22 de marzo de 2010] Disponible en: http://diccionario.sensagent.com/reconocimiento/es-es/

DICCIONARIO DE LAS CIENCIAS DE LA EDUCACIÓN. (1995). Madrid. Editorial Santillana, S.A. 
Galaz Fontes, Jesús Francisco. (2003). La satisfacción laboral de los académicos en una universidad estatal pública: la realidad institucional bajo la lente del profesorado. México. Asociación Nacional de Universidades e Instituciones de Educación Superior (ANUIES)

Hernández-Sampieri Roberto, Baptista, Lucio Pilar y Fernández-Collado, Carlos. (2008). Metodología de la investigación. $4^{\circ}$ Edición. México: McGraw-Hill Interamericana.

Mankeliunas, Mateo V. (1996). Psicología de la motivación. $2^{\circ}$ Edición. México. Editorial Trillas.

Manual de la Educación. (2000). Barcelona: Océano Grupo Editorial, S.A.

Martínez Selva, José María (2004). Estrés Laboral para directivos y empleados. Madrid. Rigorma Gráfica, S.A.

Murillo Moronta, Iraiza J. Nivel de satisfacción del personal académico del Instituto Pedagógico de Miranda José Manuel Siso Martínez en relación con el estilo de liderazgo del Jefe del Departamento...Sapiens [en línea] 2006, 7 (junio): [fecha de consulta: 5 de junio de 2009] Disponible en: $\leq$ http://redalyc.uaemex.mx/redalyc/src/ inicio/ArtPdfRed.jsp?iCve=41070104> ISSN 1317-5815

Ramos Madrigal, Abel. El compromiso organizacional y su relación con el desempeño docente de los profesores del programa universitario de inglés de la Universidad Colima (tesis doctoral) [fecha de consulta: 17 de junio de 2009] Disponible en: http://digeset.ucol.mx/tesis_posgrado/resumen.php?ID=1532

Rico, Rubén Roberto. (1996). Total customer satisfacción (satisfacción y deleite total de los clientes): vías de planificación y acción para crear, administrar y medir la satisfacción y el deleite de los clientes. Buenos Aires. Macchi Grupo Editor S.A.

Rosales Villarroel, Pedro Enrique. (2007). Organización Educativa - Apuntes de Docencia. Talca - Chile.

Vaillant, Denise y Rossel Cecilia. (2006). Maestros de escuelas básicas en América Latina: hacia una radiografía de la profesión. Santiago. Editorial San Marino

Zabalza, Miguel A. (1995). Diseño y Desarrollo Curricular. $6^{\circ}$ Edición. Madrid. Ediciones Narcea, S.A. 